\title{
The Prognostic Role of Mitotic Index in Hepatocellular Carcinoma Patients after Curative Hepatectomy
}

\author{
Sang Yun Ha, MD, PhD \\ Misun Choi, MD \\ Taebum Lee, MD \\ Cheol-Keun Park, MD, PhD
}

Department of Pathology and Translational Genomics, Samsung Medical Center,

Sungkyunkwan University

School of Medicine, Seoul, Korea
Correspondence: Cheol-Keun Park, MD, PhD Department of Pathology

and Translational Genomics,

Samsung Medical Center,

Sungkyunkwan University School of Medicine,

81 Irwon-ro, Gangnam-gu, Seoul 06351, Korea

Tel: 82-2-3410-2766

Fax: 82-2-3410-0025

E-mail: ckpark@skku.edu

Received November 11, 2014

Accepted February 6, 2015

Published Online March 18, 2015

\begin{abstract}
Purpose
High proliferation rate is a hallmark of cancer. The mitotic index is a useful and simple method for analysis of cell proliferation. However, the practical utility of mitotic index as a predictor of prognosis in patients with hepatocellular carcinoma ( $\mathrm{HCC}$ ) has not been determined. Therefore, we examined mitotic index as a prognostic marker in $\mathrm{HCC}$ patients.
\end{abstract}

\section{Materials and Methods}

We counted the number of mitotic cells in 10 high-power fields of the tumor area on hematoxylin and eosin-stained slides representing 282 surgically resected HCCs. The highest number of mitotic cells was defined as the mitotic index.

\section{Results}

High mitotic index was observed in 127 of 282 HCCs. High mitotic index showed significant association with younger age, larger tumor size, higher Edmondson grade, microvascular invasion, major portal vein invasion, intrahepatic metastasis, higher American Joint Committee on Cancer (AJCC) T-stage, higher Barcelona Clinic Liver Cancer (BCLC) stage, higher alpha-fetoprotein level, hepatitis B virus etiology, and liver cirrhosis. Patients with high mitotic index had shorter disease-specific survival (DSS) $(p<0.001)$ and tended to have shorter recurrence-free survival $(p=0.112)$. In subgroup analysis among patients with a larger tumor size, microvascular invasion, intrahepatic metastasis, higher AJCC T-stage, and higher BLCL stage, high mitotic index showed unfavorable influences on DSS $(p=0.001, p=0.008$, $p=0.003, p=0.012$, and $p<0.001$, respectively). In addition, high mitotic index was an independent predictor of shorter DSS $(p=0.004)$.

\section{Conclusion}

High mitotic index may be a novel predictor of DSS in patients with HCC and may have utility as an auxiliary prognostic factor in HCC.

\section{Introduction}

Surgically treated patients with hepatocellular carcinoma (HCC), which represent a highly selected group, have higher survival rates compared to those of medically treated patients at a comparable stage [1]. However, long-term prognosis remains unsatisfactory because of the high incidence of tumor recurrence and metastasis after hepatectomy $[2,3]$.
Key words

Mitotic index, Prognostic factor, Survival, Hepatocellular carcinoma
Thus, identification of markers of poor prognosis is important in order to provide the opportunity for timely intervention.

High proliferation rate, a classic hallmark of cancer, is due to the self-sufficiency of growth signals, insensitivity to antigrowth signals, and limitless replicative potential [4]. A variety of methods, including analysis of proliferating cell nuclear antigen, bromodeoxyuridine, argyrophilic nuclear organized regions, Ki-67 nuclear antigen, and phosphory- 
lated histone $\mathrm{H} 3$, are used in evaluation of proliferative activity [5-7]. However, many of these methods cannot be applied in daily clinical practice [5]. In contrast, the mitotic index, which is a useful and simple method for analysis of cell proliferation, can be easily applied to routine clinical practice [5]. The prognostic role of mitotic index in patient survival has been confirmed in several cancers. The mitotic index is used for tumor grading of breast and ovarian cancers $[8,9]$. In addition, mitotic index has been incorporated in the American Joint Committee on Cancer (AJCC) seventh tumor staging system for malignant melanoma, gastrointestinal tumor, and neuroendocrine tumors of the gastrointestinal tract [10]. In HCCs, previous studies indicated a potential role of high mitotic index as an adverse prognostic indicator in cohorts of fewer than 200 patients [5,6,11,12]. However, the practical utility of mitotic index as a predictor of prognosis in patients with HCC has not been determined.

In this study, we evaluated mitotic index as a possible prognostic marker in a large cohort of 282 patients with primary HCC who received long-term follow-up for 120 months. We also attempted to determine the cutoff value for mitotic index that showed the most significant prognostic role in HCC patients.

\section{Materials and Methods}

\section{Patients}

A total of 290 patients who were pathologically confirmed to have primary HCC and underwent curative resection at Samsung Medical Center, Seoul, Korea between July 2000 and May 2006 were enrolled in this study. Eight patients who received preoperative treatments, including transcatheter arterial chemoembolization, radiofrequency ablation, and radiation therapy, were excluded; therefore, 282 patients were included in this study. Curative resection was defined as complete resection of all tumor nodules with clear microscopic resection margins and no residual tumors on computed tomography scans performed 1 month after surgery. All patients had Child-Pugh A liver function. This study was approved by the Institutional Review Board of Samsung Medical Center.

Clinical parameters, including age, gender, date of surgery, serum $\alpha$-fetoprotein (AFP), and serum albumin, were obtained by reviewing the medical records. Paraffin-embedded tissues were sectioned and stained with hematoxylin and eosin. When the tumor was less than $3 \mathrm{~cm}$ in size, all tumors were sectioned and embedded. When the tumor was larger than $3 \mathrm{~cm}$ in size, at least four sections were taken for the pathologic examinations and the mean number of blocks was one for $1 \mathrm{~cm}$ of tumor diameter. Histopathologic features of HCCs, including histologic differentiation, microvascular invasion, major portal vein invasion, intrahepatic metastasis, multicentric occurrence, and non-tumor liver pathology, were reviewed by two pathologists (S.Y.H. and C.-K.P.). Tumor differentiation was defined according to the criteria of Edmondson and Steiner [13]. Intrahepatic metastasis and multicentric occurrence were determined according to the criteria of the Liver Cancer Study Group of Japan [14]. Multicentric HCCs are classified as either synchronous or metachronous. HCC recurrence within the first two years following surgery is mainly due to intrahepatic metastasis, whereas late recurrence usually results from multicentric occurrence [15]. Using 2 years as a cutoff, tumor recurrence was classified as either early recurrence or late recurrence [16]. All patients were staged according to the AJCC staging system [10] and Barcelona Clinic Liver Cancer (BCLC) staging classification [17].

During follow-up, serum AFP levels were monitored and three phase dynamic computed tomography scan or magnetic resonance imaging was performed every 3 months after surgery. The median follow-up period was 120 months (range, 14 to 151 months) for survivors. Recurrence-free survival (RFS) was measured from the date of surgery until detection of tumor recurrence. Disease-specific survival (DSS) was defined as the interval between the date of surgery and the date of HCC-related death, which was defined as: (1) the tumor occupying more than $80 \%$ of the liver, (2) portal venous tumor thrombus proximal to the second bifurcation, (3) obstructive jaundice due to the tumor, (4) distant metastases, and (5) variceal hemorrhage with portal venous tumor thrombus proximal to the first bifurcation [18].

\section{Evaluation of mitotic index}

Two liver pathologists (S.Y.H. and C.-K.P.) counted the number of mitotic cells in 10 high-power fields (HPFs) of hematoxylin and eosin-stained slides, and found areas containing the most mitotic figures, the so-called hot spot. After counting the mitoses in the hot spot, the count was extended to adjacent non-overlapping fields. If no hot spot could be found and mitoses were sparse and randomly scattered throughout the lesion, a representative mitosis was chosen and the count began with that field and was extended to adjacent fields. According to the criteria of mitotic figures defined by Baak [19], mitotic cells were counted only if there was complete concordance between the two pathologists using a multi-head microscope. The highest number of mitotic cells was defined as the mitotic index. The X-Tile statistics package (Yale University, New Haven, CT) was used to determine the optimal cutoff value with the highest 

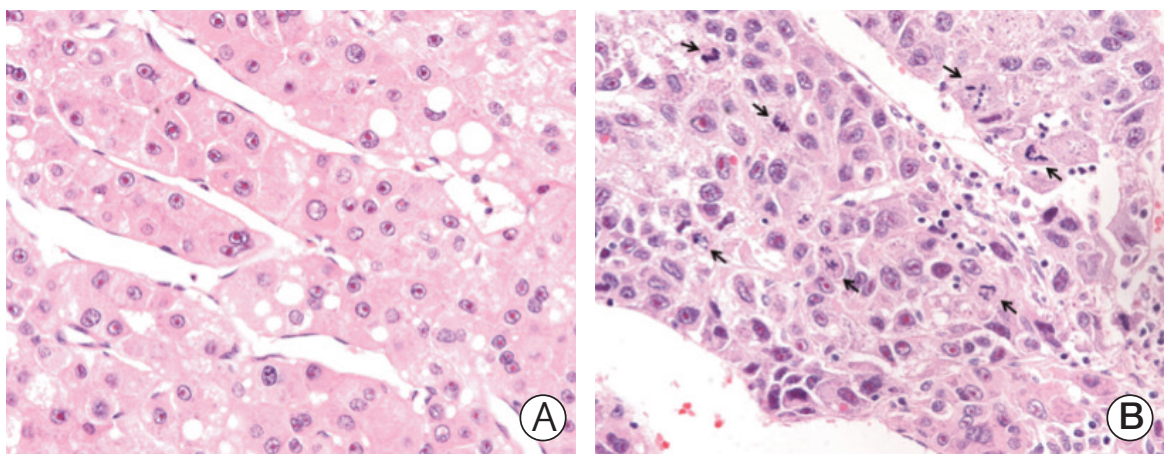

Fig. 1. Microscopic features of hepatocellular carcinomas. (A) A case of low mitotic index showing no mitotic cells. (B) A case of high mitotic index showing many mitotic cells (arrows) (A and B, H\&E staining, $\times 400$ ).

level of statistical significance related to patient survival [20]; cases were dichotomized into low and high mitotic index groups based on the established cutoff value.

\section{Statistical analysis}

Analysis of the association between mitotic index and clinicopathologic parameters was performed using the chi-square test, Fisher exact test, or Cochran Armitage test. Survival analysis was performed using the Kaplan-Meier method. Differences in survival rates were assessed using the log-rank test or Breslow test. The Cox proportional hazard regression model was used to assess the association between clinicopathologic factors and survival time. Significant prognostic factors identified by univariate analysis were entered into multivariate analysis. We examined the proportional hazard assumption graphically to determine whether variables in the Cox proportional hazard model were constants that do not depend on time. All statistical analyses were performed using SPSS ver. 18 software (SPSS Inc., Chicago, $\mathrm{IL})$ or R software (ver. 3.03); $\mathrm{p}$-values less than 0.05 were considered statistically significant.

\section{Results}

\section{Clinicopathologic features of patients}

The median patient age was 53 years (range, 17 to 76 years); 234 patients were males, and 48 were females. Two hundred and eighteen patients $(77.3 \%)$ were infected with hepatitis B virus, and $26(9.2 \%)$ with hepatitis C virus. No viral marker was recognized in 38 patients (13.5\%). Two hun- dred and three patients $(72.0 \%)$ suffered from tumor recurrence; 153 patients $(54.3 \%)$ from early recurrence, and 50 patients $(17.7 \%)$ from late recurrence. Ninety-eight patients $(34.8 \%)$ died of HCC. Twenty-nine of the 127 deaths were due to non-HCC-related causes. Seventeen of the 29 deaths were due to hepatic failure; eight were due to non-hepatic causes, and four were due to unknown causes.

\section{Mitotic index in HCC}

The mean mitotic index was 7.75 (95\% confidence interval, 6.47 to 9.03 ) and the median value was 3.00 (range, 0 to 60). Using the X-tile package, mitotic index was graded as low (4 or less mitoses per $10 \mathrm{HPFs}$ ) or high ( 5 or more mitoses per 10 HPFs) (Fig. 1). High mitotic index was observed in 127 of the 282 HCCs (45.0\%).

Associations between mitotic index and clinicopathologic parameters are summarized in Table 1. High mitotic index showed significant association with younger age $(\mathrm{p}<0.001)$, larger tumor size $(\mathrm{p}=0.022)$, higher Edmondson grade $(\mathrm{p}<$ $0.001)$, microvascular invasion $(p<0.001)$, major portal vein invasion $(\mathrm{p}=0.026)$, intrahepatic metastasis $(\mathrm{p}<0.001)$, higher AFP level $(p<0.001)$, hepatitis B virus etiology $(p=0.012)$, and liver cirrhosis $(\mathrm{p}=0.016)$. As the AJCC T-stage or BCLC stage increased, the frequency of high mitotic index also showed a significant increase $(p<0.001$ and $p<0.001$, respectively). High mitotic index did not show correlation with early or late recurrences.

\section{Effect of mitotic index on patient survival}

The 3-, 5-, 7-, and 9-year RFS rates for 282 HCC patients were $43.5 \%, 37.2 \%, 31.1 \%$, and $30.0 \%$, respectively. The $3-, 5-$, 7-, and 9-year DSS rates were $75.1 \%, 67.1 \%, 59.6 \%$, and $53.4 \%$, respectively. Patients with high mitotic index had shorter 
Table 1. Association between mitotic index and clinicopathologic parameters in 282 hepatocellular carcinomas

\begin{tabular}{|c|c|c|c|c|}
\hline \multirow{2}{*}{ Variable } & \multirow{2}{*}{ No. } & Low mitotic index & High mitotic index & \multirow{2}{*}{ p-value } \\
\hline & & $\leq 4 / 10$ & $\geq 5 / 10 \mathrm{HPF}$ & \\
\hline \multicolumn{5}{|l|}{ Age (yr) } \\
\hline$\leq 55$ & 163 & $75(46.0)$ & $88(54.0)$ & \multirow{2}{*}{$<0.001$} \\
\hline$>55$ & 119 & $80(67.2)$ & $39(32.8)$ & \\
\hline \multicolumn{5}{|l|}{ Sex } \\
\hline Female & 48 & $22(45.8)$ & $26(54.2)$ & \multirow[t]{2}{*}{0.163} \\
\hline Male & 234 & $133(56.8)$ & $101(43.2)$ & \\
\hline \multicolumn{5}{|c|}{ Tumor size $(\mathrm{cm})$} \\
\hline$\leq 5.0$ & 176 & $106(60.2)$ & $70(39.8)$ & \multirow[t]{2}{*}{0.022} \\
\hline$>5.0$ & 106 & $49(46.2)$ & $57(53.8)$ & \\
\hline \multicolumn{5}{|c|}{ Edmondson grade } \\
\hline I & 32 & $28(87.5)$ & $4(12.5)$ & \multirow[t]{3}{*}{$<0.001$} \\
\hline II & 226 & 125 & $101(44.7)$ & \\
\hline III & 24 & $2(8.3)$ & $22(91.7)$ & \\
\hline \multicolumn{5}{|c|}{ Microvascular invasion } \\
\hline$(-)$ & 130 & $99(76.2)$ & $31(23.8)$ & \multirow{2}{*}{$<0.001$} \\
\hline$(+)$ & 152 & $56(36.8)$ & $96(63.2)$ & \\
\hline \multicolumn{5}{|c|}{ Major portal vein invasion } \\
\hline$(-)$ & 271 & $153(56.5)$ & $118(43.5)$ & \multirow[t]{2}{*}{$0.026^{\mathrm{a}}$} \\
\hline$(+)$ & 11 & $2(18.2)$ & $9(81.8)$ & \\
\hline \multicolumn{5}{|c|}{ Intrahepatic metastasis } \\
\hline$(-)$ & 218 & $132(60.6)$ & $86(39.4)$ & \multirow{2}{*}{0.001} \\
\hline$(+)$ & 64 & $23(35.9)$ & $41(64.1)$ & \\
\hline \multicolumn{5}{|c|}{ Multicentric occurrence } \\
\hline$(-)$ & 265 & $144(54.3)$ & $121(45.7)$ & \multirow[t]{2}{*}{0.405} \\
\hline$(+)$ & 17 & $11(64.7)$ & $6(35.3)$ & \\
\hline \multicolumn{5}{|c|}{ AJCC T-stage } \\
\hline 1 & 122 & $93(76.2)$ & $29(23.8)$ & \multirow[t]{4}{*}{$\left.<0.001^{\mathrm{b}}\right)$} \\
\hline 2 & 112 & $45(40.2)$ & $67(59.8)$ & \\
\hline 3 & 42 & $16(38.1)$ & $26(61.9)$ & \\
\hline 4 & 6 & $1(16.7)$ & $5(83.3)$ & \\
\hline BCLC stage & & & & \\
\hline $0-\mathrm{A}$ & 162 & $101(62.3)$ & $61(37.7)$ & $<0.001^{\text {b) }}$ \\
\hline B & 107 & $51(47.7)$ & $56(52.3)$ & \\
\hline $\mathrm{C}$ & 13 & $3(23.1)$ & $10(76.9)$ & \\
\hline Albumin le & & & & \\
\hline$>3.5$ & 261 & $144(55.2)$ & $117(44.8)$ & 0.805 \\
\hline$\leq 3.5$ & 21 & $11(52.4)$ & $10(47.6)$ & \\
\hline AFP level & & & & \\
\hline$\leq 200$ & 172 & $109(63.4)$ & $63(36.6)$ & $<0.001$ \\
\hline$>200$ & 110 & $46(41.8)$ & $64(58.2)$ & \\
\hline Etiology & & & & \\
\hline Non-viral & 38 & $23(60.5)$ & $15(39.5)$ & 0.012 \\
\hline $\mathrm{HBV}$ & 218 & $111(50.9)$ & $107(49.1)$ & \\
\hline $\mathrm{HCV}$ & 26 & $21(80.8)$ & $5(19.2)$ & \\
\hline Liver cirrho & & & & \\
\hline$(-)$ & 140 & $87(62.1)$ & $53(37.9)$ & 0.016 \\
\hline$(+)$ & 142 & $68(47.9)$ & 74 (52.1) & \\
\hline Early recur & & & & \\
\hline$(-)$ & 129 & $75(58.1)$ & 54 (41.9) & 0.325 \\
\hline$(+)$ & 153 & $80(52.3)$ & $73(47.7)$ & \\
\hline
\end{tabular}


Table 1. Continued

$\begin{array}{lccc}\text { Variable } & \text { No. } & \text { Low mitotic index } & \frac{\text { High mitotic index }}{\geq 5 / 10 ~ H P F} \\ \end{array}$

Values are presented as number (\%). AJCC, American Joint Committee on Cancer; BCLC, Barcelona Clinic Liver Cancer; AFP, $\alpha$-fetoprotein; HBV, hepatitis B virus; HCV, hepatitis C virus. ${ }^{a}$ By Fisher's exact test, ${ }^{b / B y}$ Cochran-Armitage test, otherwise by chi-square test, ${ }^{\mathrm{c}}$ No early or late recurrence.
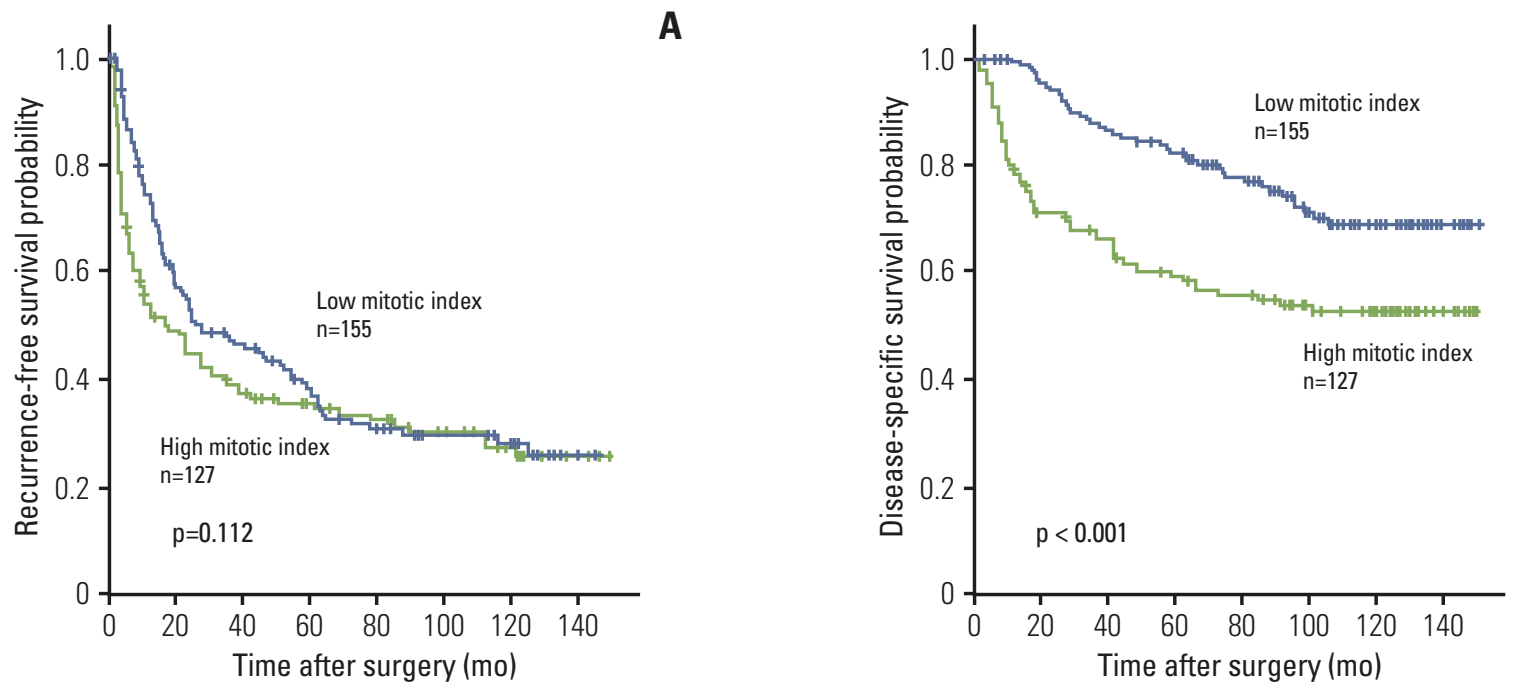

Fig. 2. Kaplan-Meier survival curves by log-rank test showing recurrence-free survival (A) and disease-specific survival (B) according to the mitotic index in 282 hepatocellular carcinomas.

DSS $(p<0.001)$ and tended to have shorter RFS $(p=0.112)$ (Fig. 2). By applying the Breslow test, which attributes greater weight to earlier events, patients with high mitotic index had shorter RFS ( $p=0.003)$. In subgroup analysis among patients with a larger tumor size $(>5 \mathrm{~cm})$, microvascular invasion, intrahepatic metastasis, higher AJCC T-stage, and higher BLCL stage, high mitotic index showed unfavorable influences on DSS ( $\mathrm{p}=0.001, \mathrm{p}=0.008, \mathrm{p}=0.003, \mathrm{p}=0.012$, and $\mathrm{p}<0.001$, respectively) (Fig. 3).

In univariate analysis, larger tumor size, Edmondson grade III, microvascular invasion, major portal vein invasion, intrahepatic metastasis, higher AJCC T-stage, higher BCLC stage, lower albumin level, and higher AFP level showed unfavorable influences on both RFS and DSS. Viral etiology showed an unfavorable influence on RFS. In addition, we found that high mitotic index showed an unfavorable influ- ence on DSS $(\mathrm{p}<0.001)$ (Table 2).

Because AJCC T-stage and BCLC stage were associated with vascular invasion, to avoid potential bias we did not perform multiple analyses using these indices. In multivariate analysis, intrahepatic metastasis and lower albumin level were found to be independent predictors of both shorter RFS and shorter DSS. Higher AFP level was found to be an independent predictor of shorter RFS. Larger tumor size and high mitotic index $(\mathrm{p}=0.004)$ were found to be independent predictors of shorter DSS. Patients with high mitotic index were more likely to suffer from disease-specific death compared to those with low mitotic index (hazard ratio, 1.818) (Table 3). Multivariate analysis of RFS with mitotic index could not be performed, because the survival curves according to mitotic index were crossed and the proportional hazard assumption was violated. 
Size $\leq 5 \mathrm{~cm}$

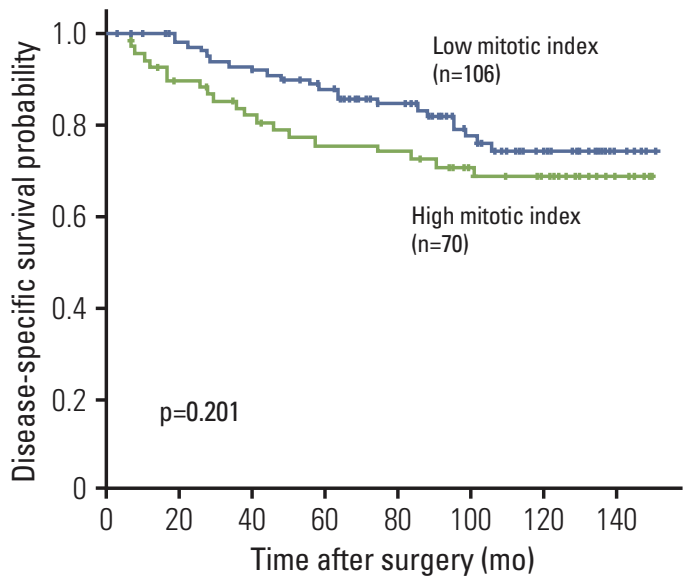

Microvascular invasion (-)

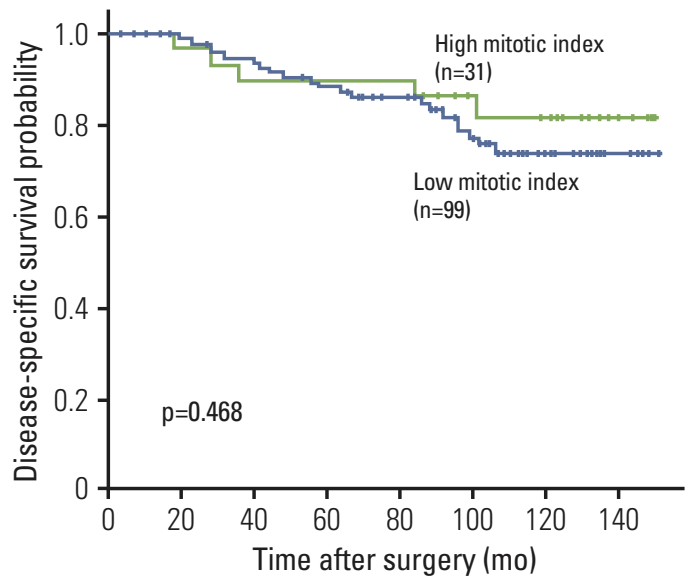

Intrahepatic metastasis (-)

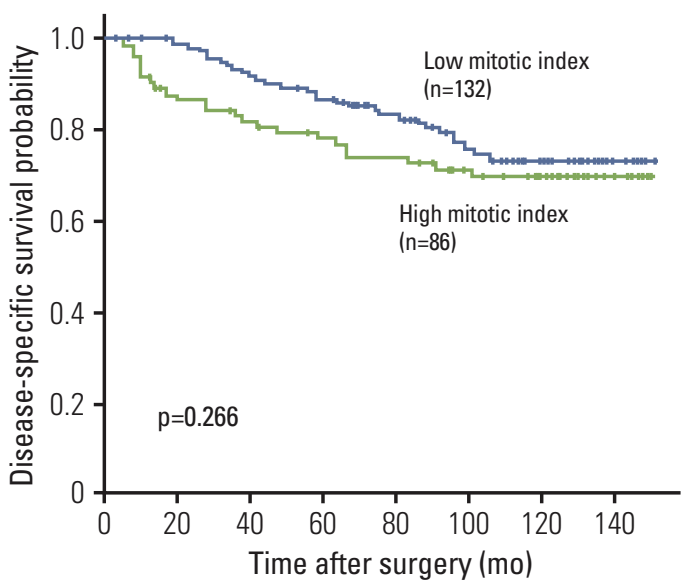

C

E
Size $>5 \mathrm{~cm}$

B

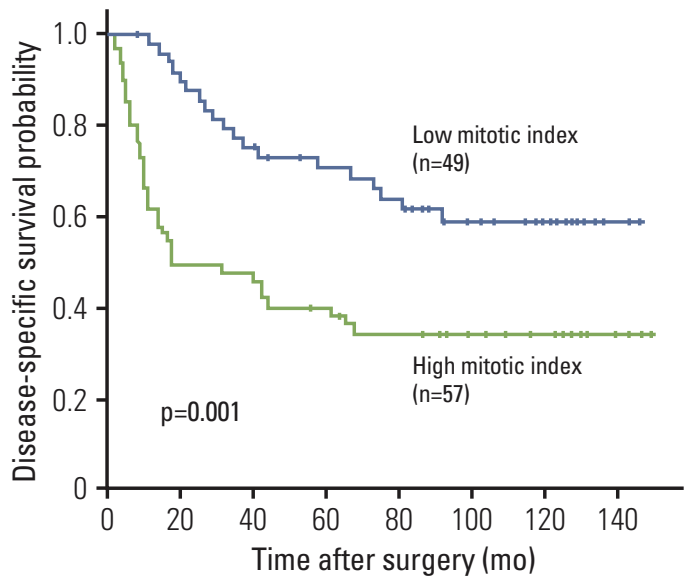

Microvascular invasion (t)

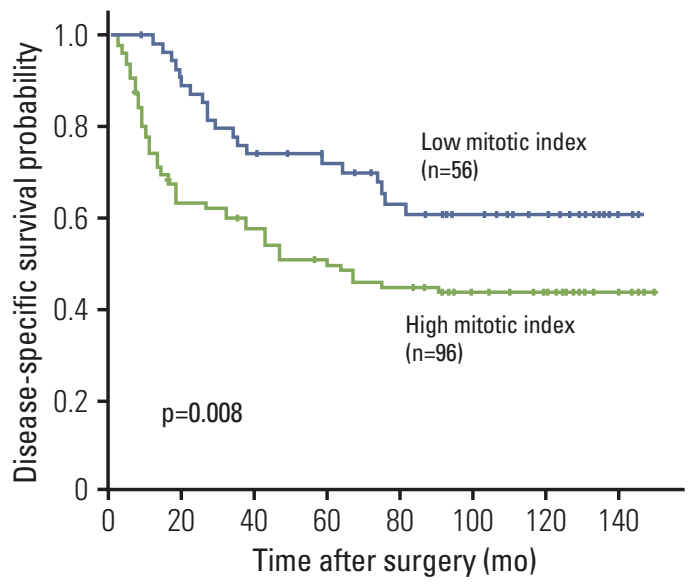

Intrahepatic metastasis (+)

$\mathbf{F}$

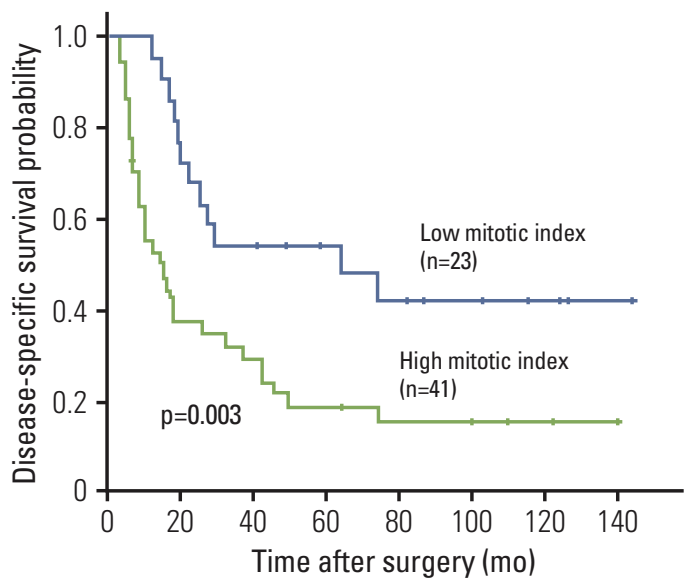

Fig. 3. Kaplan-Meier survival curves by log-rank test showing disease-specific survival according to the mitotic index in subgroups by tumor size $(\mathrm{A}, \mathrm{B})$, microvascular invasion $(\mathrm{C}, \mathrm{D})$, intrahepatic metastasis $(\mathrm{E}, \mathrm{F})$. (Continued to the next page) 
AJCC T-stage 1

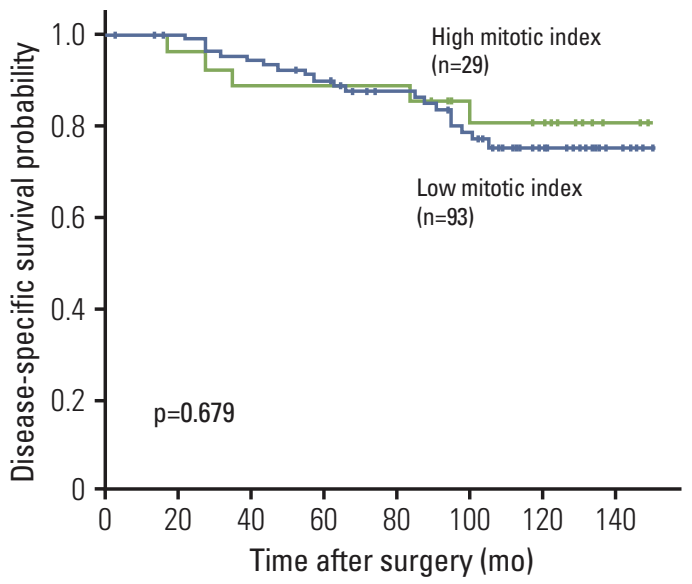

BCLC stage $0, A$

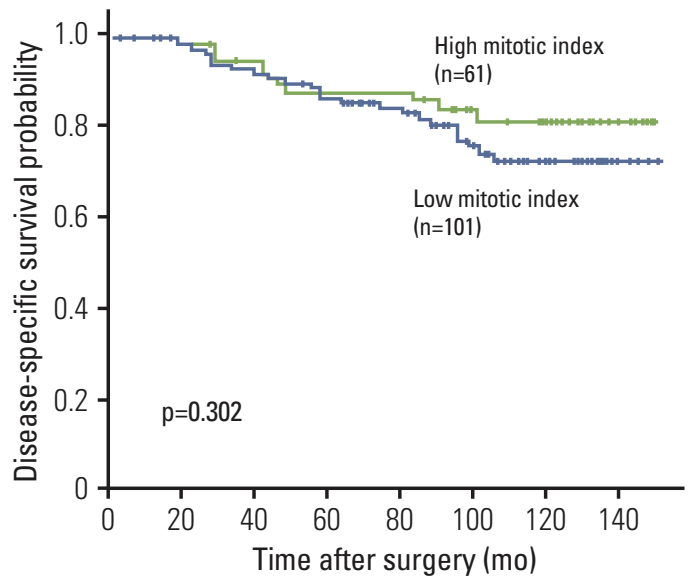

AJCC T-stage 2-4

H

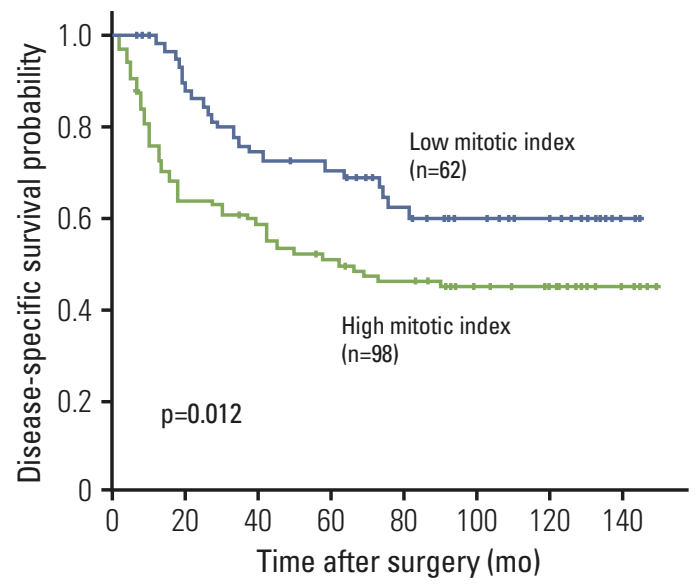

BCLC stage $B, C$

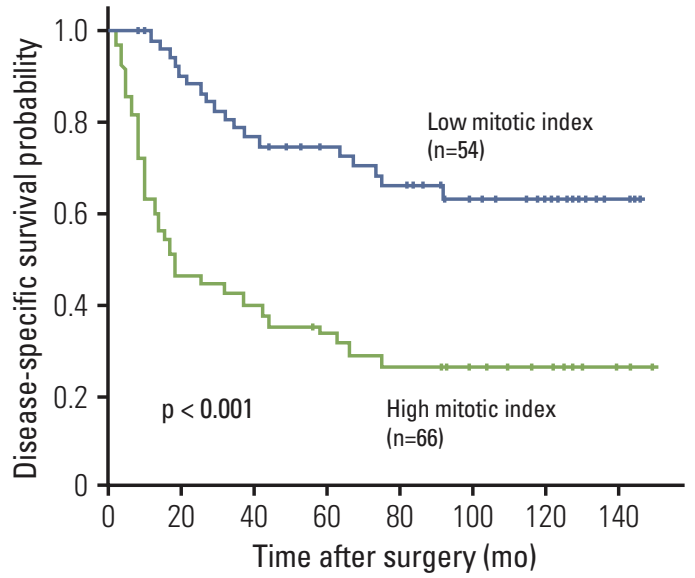

Fig. 3. (Continued from the previous page) American Joint Committee on Cancer (AJCC) T-stage (G, H), and Barcelona Clinic Liver Cancer (BCLC) stage (I, J).

\section{Discussion}

In the current study, we elucidated the prognostic significance of mitotic index in a large cohort of patients with primary HCC who received long-term follow-up. We attempted to establish a cutoff value for mitotic index that showed the most significant prognostic role in HCC patients. Low mitotic index was defined as four or fewer mitoses per $10 \mathrm{HPFs}$, and high mitotic index as five or more. High mitotic index showed correlation with larger tumor size, higher Edmondson grade, microvascular invasion, major portal invasion, intrahepatic metastasis, higher AJCC T-stage, higher BCLC stage, higher AFP level, hepatitis B virus etiology, and liver cirrhosis. In addition, patients with high mitotic index had shorter DSS and tended to have shorter RFS. The mean time gap between recurrence and death in the high mitotic index group was significantly lower than in the low mitotic index group (30.5 months vs. 43.8 months, $\mathrm{p}=0.004$ ). Treatment modality after recurrence did not differ statistically between low versus high mitotic index group $(\mathrm{p}=0.38)$ (Supplementary Table 1). It is assumed that the high mitotic index group had poorer response to the salvage treatment after recurrence than the low mitotic index group.

Only a few studies on the prognostic role of mitotic index in HCC have been reported. Haratake et al. [11], who divided 
Table 2. Univariate analyses of recurrence-free survival and disease-specific survival in 282 hepatocellular carcinomas

\begin{tabular}{|c|c|c|c|c|}
\hline \multirow{2}{*}{ Variable } & \multicolumn{2}{|c|}{ Recurrence-free survival } & \multicolumn{2}{|c|}{ Disease-specific survival } \\
\hline & HR $(95 \% \mathrm{CI})$ & p-value & HR $(95 \%$ CI) & p-value \\
\hline Age ( $>55$ yr vs. $\leq 55$ yr $)$ & $0.968(0.723-1.297)$ & 0.829 & $0.932(0.623-1.394)$ & 0.731 \\
\hline Sex (male vs. female) & $0.989(0.822-1.191)$ & 0.910 & $0.825(0.483-1.410)$ & 0.481 \\
\hline Tumor size $(>5.0 \mathrm{~cm}$ vs. $\leq 5.0 \mathrm{~cm})$ & $1.663(1.244-2.223)$ & 0.001 & $2.981(1.996-4.453)$ & $<0.001$ \\
\hline Edmondson grade (III vs. I, II) & $2.242(1.421-3.538)$ & 0.001 & $2.662(1.509-4.697)$ & 0.001 \\
\hline Microvascular invasion (+ vs. - ) & $2.210(1.644-2.971)$ & $<0.001$ & $3.241(2.067-5.080)$ & $<0.001$ \\
\hline Major portal vein invasion (+ vs. -) & $3.420(1.801-6.492)$ & $<0.001$ & $5.008(2.510-9.994)$ & $<0.001$ \\
\hline Intrahepatic metastasis (+ vs. -) & $4.980(3.593-6.903)$ & $<0.001$ & $5.820(3.878-8.734)$ & $<0.001$ \\
\hline Multicentric occurrence (+ vs. - ) & $1.183(0.625-2.241)$ & 0.606 & $0.668(0.245-1.816)$ & 0.429 \\
\hline AJCC T-stage (2, 3, 4 vs. 1$)$ & $4.059(2.866-5.748)$ & $<0.001$ & $5.197(3.414-7.911)$ & $<0.001$ \\
\hline BCLC stage $(\mathrm{B}, \mathrm{C}$ vs. $0, \mathrm{~A})$ & $2.141(1.605-2.855)$ & $<0.001$ & $3.955(2.596-6.024)$ & $<0.001$ \\
\hline Albumin level $(\leq 3.5 \mathrm{~g} / \mathrm{dL}$ vs. $>3.5 \mathrm{~g} / \mathrm{dL})$ & $2.159(1.289-3.616)$ & 0.003 & $3.042(1.687-5.485)$ & $<0.001$ \\
\hline AFP level (> $200 \mathrm{ng} / \mathrm{mL}$ vs. $\leq 200 \mathrm{ng} / \mathrm{mL}$ ) & $1.705(1.279-2.272)$ & $<0.001$ & $1.769(1.190-2.630)$ & 0.005 \\
\hline Etiology (viral vs. non-viral) & $2.032(1.233-3.348)$ & 0.005 & $1.530(0.795-2.944)$ & 0.203 \\
\hline Liver cirrhosis (+ vs. -) & $1.314(0.986-1.752)$ & 0.062 & $1.036(0.697-1.539)$ & 0.862 \\
\hline Mitotic index (high vs. low) & $1.261(0.946-1.680)$ & 0.113 & $2.131(1.426-3.185)$ & $<0.001$ \\
\hline
\end{tabular}

HR, hazard ratio; CI, confidence interval; AJCC, American Joint Committee on Cancer; BCLC, Barcelona Clinic Liver Cancer; AFP, $\alpha$-fetoprotein.

Table 3. Multivariate analyses of recurrence-free survival and disease-specific survival in 282 hepatocellular carcinomas

\begin{tabular}{|c|c|c|c|c|}
\hline \multirow{2}{*}{ Variable } & \multicolumn{2}{|c|}{ Recurrence-free survival } & \multicolumn{2}{|c|}{ Disease-specific survival } \\
\hline & HR $(95 \%$ CI $)$ & p-value & $\mathrm{HR}(95 \% \mathrm{CI})$ & p-value \\
\hline Tumor size $(>5.0 \mathrm{~cm}$ vs. $\leq 5.0 \mathrm{~cm})$ & $0.998(0.714-1.395)$ & 0.991 & $1.824(1.176-2.828)$ & 0.007 \\
\hline Edmondson grade (III vs. I, II) & $1.453(0.889-2.375)$ & 0.136 & $1.298(0.711-2.369)$ & 0.396 \\
\hline Microvascular invasion (+ vs. -) & $1.333(0.921-1.931)$ & 0.128 & $1.273(0.700-2.315)$ & 0.430 \\
\hline Major portal vein invasion (+ vs. -) & $0.719(0.351-1.473)$ & 0.368 & $1.040(0.489-2.212)$ & 0.918 \\
\hline Intrahepatic metastasis (+ vs. - ) & $3.615(2.382-5.487)$ & $<0.001$ & $4.196(2.696-6.530)$ & $<0.001$ \\
\hline Albumin level $(\leq 3.5 \mathrm{~g} / \mathrm{dL}$ vs. $>3.5 \mathrm{~g} / \mathrm{dL})$ & $2.095(1.189-3.693)$ & 0.011 & $2.369(1.292-4.344)$ & 0.005 \\
\hline AFP level (> $200 \mathrm{ng} / \mathrm{mL}$ vs. $\leq 200 \mathrm{ng} / \mathrm{mL}$ ) & $1.368(1.003-1.866)$ & 0.048 & $1.111(0.715-1.727)$ & 0.640 \\
\hline Etiology (viral vs. non-viral) & $1.644(0.990-2.731)$ & 0.055 & - & - \\
\hline Mitotic index (high vs. low) & - & - & $1.818(1.210-2.731)$ & 0.004 \\
\hline
\end{tabular}

$\mathrm{HR}$, hazard ratio; CI, confidence interval; AFP, $\alpha$-fetoprotein.

mitotic index into three groups (0-4/10 HPFs, 5-9/10 HPFs, 10 and more/10 HPFs), observed a better prognosis for patients with HCC who exhibited low mitotic activity. They reported that longer survival periods were observed in some patients with larger tumors if the mitotic indices were low. Ouchi et al. [5] showed that mitotic index was an independent significant variable, influencing the overall survival of 40 patients with HCC following hepatic resection. In addition, they found that HCC with a high mitotic index ( $\geq 5 / 10 \mathrm{HPFs}$ ) was associated with multiple tumors and advanced tumor stage. They also reported a highly significant correlation between mitotic index and Ki-67 labelling index $(\mathrm{r}=0.870)$. Nanashima et al. [12] also reported correlation of high mitotic index ( $\geq 5 / 10 \mathrm{HPFs}$ ) with overall survival in 81 patients with HCC by univariate analysis. They also found a significant association of high mitotic index with vascular invasion, poor histologic differentiation, and recurrence rate. Our findings are consistent with those of previous studies. Interestingly, the criteria of mitotic index used in previous studies are the same as those applied in our study, which were determined statistically for the strongest prognostic effect. To the best of our knowledge, this is the first report to show 
that high mitotic index is an independent predictor of shorter DSS. However, high mitotic index was not an independent predictor of shorter RFS. HCC recurrence within the first 2 years following surgery is mainly due to intrahepatic metastasis, whereas recurrence after 2 years following surgery usually results from multicentric occurrence of HCC [15]. It is assumed that the high mitotic index group did not show shorter RFS after 48 months following surgery, because recurrence after 48 months following surgery was mainly due to newly developed HCC.

The most outstanding finding of this study is that subgroup analysis among patients with larger tumor size, microvascular invasion, intrahepatic metastasis, higher AJCC T-stage, and higher BLCL stage showed unfavorable influences of high mitotic index on DSS. Counting mitotic cells is a simple, rapid, and inexpensive method which can be routinely performed in daily practice. Our results suggest that mitotic index is a new auxiliary prognostic factor in HCC.

\section{Conclusion}

Our data show, for the first time, that high mitotic index is an independent predictor of shorter DSS in surgically resected HCCs. Mitotic index could be used as an auxiliary prognostic factor in patients with HCC. Prospective studies are needed to further investigate the role of mitotic index as a prognostic factor in HCC.

\section{Electronic Supplementary Material}

Supplementary materials are available at Cancer Research and Treatment website (http://www.e-crt.org).

\section{Conflicts of Interest}

Conflict of interest relevant to this article was not reported.

\section{Acknowledgments}

This study was supported by Samsung Biomedical Research Institute Grant (\#SMX 1131851).

\section{References}

1. Okuda K, Ohtsuki T, Obata H, Tomimatsu M, Okazaki N, Hasegawa $\mathrm{H}$, et al. Natural history of hepatocellular carcinoma and prognosis in relation to treatment. Study of 850 patients. Cancer. 1985;56:918-28.

2. Llovet JM, Schwartz M, Mazzaferro V. Resection and liver transplantation for hepatocellular carcinoma. Semin Liver Dis. 2005;25:181-200.

3. Poon RT. Prevention of recurrence after resection of hepatocellular carcinoma: a daunting challenge. Hepatology. 2011;54: 757-9.

4. Hanahan D, Weinberg RA. The hallmarks of cancer. Cell. 2000;100:57-70.

5. Ouchi K, Sugawara T, Ono H, Fujiya T, Kamiyama Y, Kakugawa $Y$, et al. Mitotic index is the best predictive factor for survival of patients with resected hepatocellular carcinoma. Dig Surg. 2000;17:42-8.

6. Tannapfel A, Geissler F, Kockerling F, Katalinic A, Hauss J, Wittekind C. Apoptosis and proliferation in relation to histopathological variables and prognosis in hepatocellular carcinoma. J Pathol. 1999;187:439-45.

7. Romani AA, Crafa P, Desenzani S, Graiani G, Lagrasta C,
Sianesi M, et al. The expression of HSP27 is associated with poor clinical outcome in intrahepatic cholangiocarcinoma. BMC Cancer. 2007;7:232.

8. Bloom HJ, Richardson WW. Histological grading and prognosis in breast cancer: a study of 1409 cases of which 359 have been followed for 15 years. Br J Cancer. 1957;11:359-77.

9. Vang R, Shih Ie M, Kurman RJ. Ovarian low-grade and highgrade serous carcinoma: pathogenesis, clinicopathologic and molecular biologic features, and diagnostic problems. Adv Anat Pathol. 2009;16:267-82.

10. Edge SB, Byrd DR, Compton CC, Fritz AG, Greene FL, Trotti A 3rd. AJCC cancer staging manual. 7th ed. Chicago, IL: Springer; 2010. p. 191-5.

11. Haratake J, Takeda S, Kasai T, Nakano S, Tokui N. Predictable factors for estimating prognosis of patients after resection of hepatocellular carcinoma. Cancer. 1993;72:1178-83.

12. Nanashima A, Tanaka K, Yamaguchi H, Shibasaki S, Morino $\mathrm{S}$, Yoshinaga $\mathrm{M}$, et al. Fibrosis and inflammatory activity in noncancerous tissue and mitotic index of cancer tissue in patients with hepatocellular carcinoma: relationship to clinicopathological factors and prognosis after hepatic resection. 
Dig Dis Sci. 2003;48:1517-22.

13. Edmondson HA, Steiner PE. Primary carcinoma of the liver: a study of 100 cases among 48,900 necropsies. Cancer. 1954;7: 462-503.

14. Liver Cancer Study Group of Japan. General rules for the clinical and pathological study of primary liver cancer. 2nd ed. Tokyo: Kanehara; 2003.

15. Shimada M, Hamatsu T, Yamashita Y, Rikimaru T, Taguchi K, Utsunomiya $\mathrm{T}$, et al. Characteristics of multicentric hepatocellular carcinomas: comparison with intrahepatic metastasis. World J Surg. 2001;25:991-5.

16. Imamura H, Matsuyama Y, Tanaka E, Ohkubo T, Hasegawa $\mathrm{K}$, Miyagawa S, et al. Risk factors contributing to early and late phase intrahepatic recurrence of hepatocellular carcinoma after hepatectomy. J Hepatol. 2003;38:200-7.

17. Llovet JM, Bru C, Bruix J. Prognosis of hepatocellular carcinoma: the BCLC staging classification. Semin Liver Dis. 1999; 19:329-38.

18. Hoshida Y, Villanueva A, Kobayashi M, Peix J, Chiang DY, Camargo A, et al. Gene expression in fixed tissues and outcome in hepatocellular carcinoma. N Engl J Med. 2008;359: 1995-2004.

19. Baak JP. Mitosis counting in tumors. Hum Pathol. 1990;21:683-5.

20. Camp RL, Dolled-Filhart M, Rimm DL. X-tile: a new bioinformatics tool for biomarker assessment and outcome-based cut-point optimization. Clin Cancer Res. 2004;10:7252-9. 